\title{
Bilateral salpingectomy versus bilateral partial salpingectomy during cesarean delivery
}

This article was published in the following Dove Press journal: International Journal of Women's Health

\author{
Jose R Duncan ${ }^{1,2}$ \\ Heather L Jones' \\ Stefanie O Hoffer' \\ Mauro H Schenone' \\ Giancarlo Mari' \\ 'Department of Obstetrics and \\ Gynecology, University of Tennessee \\ Health Science Center, Memphis, \\ TN, USA; ${ }^{2}$ Department of Obstetrics \\ and Gynecology, University of South \\ Florida, Tampa, FL, USA
}

Objective: To compare surgical outcomes in patients undergoing bilateral salpingectomy (salpingectomy group) with those who had partial salpingectomy (partial salpingectomy group) during cesarean delivery.

Materials and methods: A chart review from July 2015 to November 2016 was performed. We included women who had sterilization during cesarean delivery. We excluded sterilization by occlusive methods. Our primary outcomes were total operative time and a composite score of transfusion rate, internal organ injury, hospital readmission, and endometritis. Secondary outcomes included menstrual abnormalities, pelvic pain, quality of life assessment, and regrets rate. We compared these outcomes between women in the salpingectomy and partial salpingectomy groups. Chi-squared, Fisher's exact, $t$-test, and Mann-Whitney $U$ were utilized for statistical analysis where appropriate. A $P<0.05$ was considered significant.

Results: We included a total of 160 pregnancies. Of these, 41 were in the salpingectomy and 119 in the partial salpingectomy group. The median total operative time was longer for the salpingectomy group (62 [IQR 54, 71] vs 60 minutes [IQR 46, 72]; $P=0.03$ ). The composite of surgical complications $(19.5 \%$ vs $12.6 \% ; P=0.28)$ was not significantly different between our study groups. Menstrual irregularities ( $P \geq 0.99)$, quality of life ( $P \geq 0.99)$, dyspareunia $(P \geq 0.99)$, dysmenorrhea $(P=0.36)$, and regrets $(P \geq 0.99)$ were not different between groups.

Conclusion: Salpingectomy during cesarean delivery increased the median operative time by 2 minutes and may not be associated with an increased risk of surgical complications. We acknowledge the need for larger multi-center trials to corroborate our outcomes.

Keywords: opportunistic salpingectomy, ovarian cancer prevention, prophylactic salpingectomy, risk-reducing surgery, sterilization

\section{Introduction}

Ovarian cancer carries the highest mortality rate of gynecological cancers. ${ }^{1}$ Recent research demonstrates that a large proportion of epithelial ovarian cancers begins in the fimbriated portion of the fallopian tubes $;^{2-4}$ therefore, bilateral total salpingectomy has been recommended by the American Congress of Obstetricians and Gynecologists and the Society for Gynecologic Oncology as an intervention to reduce the risk of ovarian cancer, instead of the more aggressive approach of performing a bilateral salpingo-oophorectomy. ${ }^{5,6}$

More than 600,000 tubal sterilizations are performed annually in the USA and about one-third of women of reproductive age utilize this method for contraception. ${ }^{7}$ Despite evidence indicating that traditional methods of sterilization like tubal ligation may reduce the risks of ovarian cancer, ${ }^{8}$ this provides a great opportunity to consider opportunistic salpingectomy for ovarian cancer prevention.
Correspondence: Jose R Dunca

Department of Obstetrics and

Gynecology, University of South

Florida, 2 Tampa General Cir \# 605I,

Tampa, FL 33606, USA

$\mathrm{Tel}+\mathrm{I} 8132598500$

Fax + I 8132590679

Email joserduncan@yahoo.com 
Studies suggest that salpingectomy during cesarean delivery or gynecological procedures do not seem to negatively affect the ovarian reserve measured by anti-Müllerian hormone (AMH) levels. ${ }^{9}{ }^{90}$ Despite these reports, information about the safety, efficacy, and feasibility of bilateral salpingectomy during cesarean delivery remains limited.

Our objective in this study was to compare surgical outcomes of salpingectomy vs partial salpingectomy techniques at the time of cesarean delivery.

\section{Materials and methods}

The University of Tennessee Health Science Center Institutional Review Board and Regional One Health approved our study. A retrospective medical record review was conducted from July 2015 to November 2016 at Regional One Health. Our participants were selected from an operative $\log$ of sterilization procedures on labor and delivery during this time period. In our practice, we discuss contraception during prenatal care; those interested in permanent sterilization, are counseled about the risks and benefits of this option. All participants in our cohort signed informed consent for surgical sterilization at least 30 days before delivery and at the time of admission to labor and delivery. When faculty familiar with bilateral salpingectomy, were available, this procedure was offered. Three of the investigators reviewed the electronic medical records of those who underwent sterilization procedures during cesarean delivery to obtain baseline demographic information at the time of surgery, including date of surgery, age, parity, gestational age, body mass index, hematocrit before delivery, and past medical and surgical history. In our institution, hematocrit is preferred over hemoglobin as hematologic indicator. Inclusion criteria included pregnant women who underwent sterilization at the time of cesarean delivery with bilateral salpingectomy, Pomeroy, modified Pomeroy, or Parkland techniques. Exclusion criteria included history of ovarian cancer, previous chemotherapy or radiation, and those women who underwent sterilization by any other methods not included in the inclusion criteria.

We compared the surgical outcomes in women who had bilateral salpingectomy during cesarean delivery (salpingectomy group) with those who had partial salpingectomy by the Pomeroy, modified Pomeroy, or Parkland techniques (partial salpingectomy group) during cesarean delivery for sterilization. The technique for the salpingectomy in our institution has been previously described. We clamp, cut, and secure the mesosalpinx vessels starting at the fimbria ovarica moving proximal toward the interstitial portion of the tube. We consider our procedure successful if $<1 \mathrm{~cm}$ of the fallopian tube is left behind. ${ }^{11}$

Our primary outcomes were total operative time and a composite of surgical complications that included as follows: need for transfusion, internal organ injury, endometritis, postpartum hemorrhage, and hospital readmission. Total operative time was defined as minutes from the start to completion of the procedure (incision to skin closure). Postpartum hemorrhage was defined as blood loss $>1,000 \mathrm{~mL} .{ }^{12} \mathrm{~A}$ need for transfusion was recorded if at least one unit of blood was received during the hospital stay. Internal organ injury was defined as intraoperative bowel or bladder injury requiring surgical repair, and hospital readmission was recorded if the patient was admitted to the hospital up to 6 weeks after cesarean delivery. Participants for whom salpingectomy was intended but was not completed were included in the salpingectomy group for analysis.

Because the total operative time was not normally distributed, we compared our median operative time for our main results, but for clinical purposes, we calculated and compared the mean total operative time among our two groups.

We studied menstrual irregularities and measures of quality of life for our secondary outcomes. This was assessed 12-24 months after their procedure by using a standardized questionnaire via telephone by three of the authors. We attempted to contact all participants on up to four different occasions or until we obtained the required data. We included short questions with simple words to improve the assimilation of our participants. Women included in the secondary outcomes analysis, verbally consented to participate in our study that was in compliance with the office of research of the University of Tennessee Health Science Center Institutional Review Board. The questions included in the questionnaire were as follows:

1. Are your periods regular or irregular since your surgery?

2. Do you have pain during your periods since surgery?

3. Do you have painful intercourse since your surgery?

4. Do you feel your quality of life is worse after your surgery?

5. Do you have any regrets?

Chi-squared, Fischer's exact, $t$-test, and Mann-Whitney $U$ were utilized for statistical analysis where appropriate. Results were presented as median (IQR), mean $\pm \mathrm{SD}$, or frequencies (n) with percentages. A $P$-value $<0.05$ was considered statistically significant. Statistical analysis was performed with SAS/STATV14.2 software. 


\section{Results}

A total of 168 women underwent a sterilization procedure at the time of cesarean delivery at our institution during our study period. Of these, eight were excluded because they had occlusive devices placed for sterilization. Thus, 160 women were included for analysis; of these, 119 were included in the partial salpingectomy and 41 in the salpingectomy group; two women that were included in the salpingectomy group only had unilateral salpingectomy because of dense adhesions. Unfortunately, prior history of endometriosis or pelvic inflammatory disease for these participants was not available.

Among the demographics and baseline characteristics, maternal age and the presence of diabetes was greater in the salpingectomy group and the hematocrit before cesarean delivery was higher in the partial salpingectomy group (Table 1).

For our primary outcomes, the median total operative time was longer for the salpingectomy group than for the partial salpingectomy group (62, [IQR 54, 71] vs 60 minutes, [IQR 46, 72]; $P=0.03$ ). The composite of surgical complications was not statistically significantly different $(19.5 \%$ vs $12.6 \%$; OR 1.68; 95\% CI: $0.65-4.32 ; P=0.41)$ among our study groups (Table 2). Of the internal organ injuries, there were two bowel injuries and one bladder injury in the partial salpingectomy group and one bowel injury in the salpingectomy group.

The mean total operative time was 8 minutes longer in the salpingectomy group ( $71 \pm 23$ vs $63 \pm 27 ; P=0.076$ ).

For our secondary outcomes, we contacted a total of 54 participants. Six of them declined participation, thus 48 gave verbal consent and were included for this analysis (30\%).
Of these, 11 were in the salpingectomy and 37 in the partial salpingectomy group. The frequency of menstrual irregularities $(27.3 \%$ vs $27 \% ; P \geq 0.99)$, dysmenorrhea $(27.7 \%$ vs $48.6 \% ; P=0.36)$, dyspareunia (9.1\% vs $8.1 \% ; P \geq 0.99)$, quality of life dissatisfaction ( $18.2 \%$ vs $16.2 \% ; P \geq 0.99)$, and the number of women with regrets $(9.1 \%$ vs $18.9 ; P=0.80)$ were not statistically different between the salpingectomy and the partial salpingectomy group.

\section{Discussion}

In our study, the median operative time was 2 minutes longer in the salpingectomy than for those undergoing partial salpingectomy by a traditional method during cesarean delivery; however, salpingectomy during cesarean delivery was not associated with an increased risk of surgical complications.

The current literature on the subject is limited, a recent large retrospective review from a California database reported an increased utilization of salpingectomy for sterilization (including during cesarean delivery) from 2011 to $2016 .^{13}$ Despite this, there are only three randomized trials addressing the safety of salpingectomy during cesarean delivery. In one study, Ganer et al, ${ }^{9}$ did not find an increased rate of surgical complications in 46 women randomized to bilateral salpingectomy, or traditional partial salpingectomy for sterilization, at the time of cesarean delivery. Their salpingectomy procedures took 13 more minutes to complete compared with the tubal ligation procedures, and the ovarian reserve was not different among their study groups. The other two trials were published in the same journal edition.

Table I Demographics and clinical characteristics of patients undergoing bilateral salpingectomy vs traditional sterilization (controls) at the time of cesarean delivery

\begin{tabular}{|c|c|c|c|}
\hline Characteristic & $\begin{array}{l}\text { Salpingectomy } \\
\text { group }(n=4 I)\end{array}$ & $\begin{array}{l}\text { Controls } \\
(n=I 19)\end{array}$ & $P$-value \\
\hline Age (years) & $31.6 \pm 5.5$ & $29.6 \pm 4.6$ & 0.023 \\
\hline \multicolumn{4}{|l|}{ Ethnicity (n) } \\
\hline African American & 29 & 90 & 0.42 \\
\hline Caucasian & 5 & 19 & \\
\hline Hispanic & 6 & 9 & \\
\hline Other & 1 & I & \\
\hline Gestational age (weeks) & $37.3 \pm 3.1$ & $37.2 \pm 3$ & 0.85 \\
\hline Parity $(n)$ & $3(\mathrm{IQR} 2,4)$ & $3(\mathrm{IQR} 2,4)$ & 0.78 \\
\hline Body mass index $\left(\mathrm{kg} / \mathrm{m}^{2}\right)$ & $38.5 \pm 8.3$ & $36.6 \pm 10.6$ & 0.31 \\
\hline Diabetes (n) & $10(24.4 \%)$ & $9(7.2 \%)$ & 0.005 \\
\hline Hypertensive disorders (n) & $12(29.3 \%)$ & $21(16.7 \%)$ & 0.087 \\
\hline$\geq 3$ prior cesareans $(n)$ & $8(20 \%)$ & $25(20 \%)$ & 0.93 \\
\hline Prior abdominal surgeries $(\mathrm{n})$ & $2(\mathrm{IQR} \mathrm{I}, 3)$ & $2(\mathrm{IQR} \mathrm{I}, 3)$ & 0.74 \\
\hline Hematocrit before cesarean (\%) & $29.1 \pm 4.4$ & $33.5 \pm 3.3$ & 0.002 \\
\hline
\end{tabular}

Notes: Data are presented as $\mathrm{n}(\%)$, means $\pm \mathrm{SD}$, or median (quartile range); statistically significant values shown in bold. 
Table 2 Surgical outcomes between our study groups

\begin{tabular}{|c|c|c|c|c|}
\hline Surgical outcome & $\begin{array}{l}\text { Salpingectomy } \\
\text { group }(n=4 I)\end{array}$ & $\begin{array}{l}\text { Partial slpingectomy } \\
\text { group }(n=|| 9)\end{array}$ & OR $(95 \% \mathrm{Cl})$ & $P$-value \\
\hline Total operative time (minutes) & $62(54,7 I)$ & $60(46,72)$ & & 0.03 \\
\hline Composite of surgical outcomes (n) & $8(19.5 \%)$ & $15(12.6 \%)$ & $1.68(0.65-4.32)$ & 0.28 \\
\hline Postpartum hemorrhage $(n)$ & $5(12.2 \%)$ & 17 (14.3\%) & $0.83(0.26-2.34)$ & 0.97 \\
\hline Internal organ injury $(n)$ & I (2.4\%) & $3(2.5 \%)$ & $0.97(0.18-12.44)$ & $>0.99$ \\
\hline Bowel & I (2.4\%) & $2(1.7 \%)$ & & \\
\hline Bladder & - & I (0.8\%) & & \\
\hline Need for transfusion $(n)$ & $5(12 \%)$ & $10(9 \%)$ & I.5। (0.48-4.77) & 0.54 \\
\hline Endometritis $(\mathrm{n})$ & I (2.4\%) & I (0.8\%) & $2.93(0.037-233.5)$ & 0.90 \\
\hline Readmission (n) & I (2.4\%) & $4(3.2 \%)$ & $0.72(0.078-6.62)$ & 0.8 \\
\hline
\end{tabular}

Notes: Data are presented as $\mathrm{n}(\%)$ and median (quartile range); statistically significant value shown in bold.

One was from Alabama that included 40 participants in the salpingectomy and 40 in the partial salpingectomy group. The investigators did not find an increased complications rate but they required an average of 15 minutes more to perform a total salpingectomy when compared with their traditional approach. They only completed the total salpingectomy in two-thirds of their attempts and only $35 \%$ of the primary surgeons included in this study would perform a total salpingectomy during cesarean delivery as part of general practice. ${ }^{14}$ The final trial included 19 women in the total salpingectomy and 20 in the partial salpingectomy group, the median total operative time was no longer in the salpingectomy group (68 vs 60 minutes; $P=0.34$ ) without an increased rate of complications. They completed the total salpingectomy in all but one of the mothers allocated in the salpingectomy group, their procedure was performed with electrothermal bipolar tissue devices and the surgeons underwent training sessions before the procedure was performed..$^{15}$ These findings are similar to our results.

In a retrospective cohort from Israel that included 149 women, Shinar et $\mathrm{al}^{16}$ did not find an increased rate of complications in women undergoing salpingectomy during cesarean delivery, and their mean operative time periods were similar between their salpingectomies and control groups.

Other studies have shown increased operative times with salpingectomy without increased rates of complications. Mcalpine et $\mathrm{al}^{17}$ reported an operative time of 10 minutes longer in performing a salpingectomy during hysterectomy; Danis et a ${ }^{18}$ reported that 20 additional minutes were needed to complete a salpingectomy postpartum compared with a partial salpingectomy. None of these studies found an increased risk of complications in their salpingectomy group.

Only one retrospective study has reported a negative effect of salpingectomy on the ovarian reserve ${ }^{19}$; and most of the research that have assessed ovarian reserve have not found a negative effect from salpingectomy on the ovarian reserve, measured by AMH levels. ${ }^{910,20-23}$ Nevertheless, adequate data are not available about the long-term effect of total salpingectomy on ovarian function.

Although the occurrence of menstrual irregularities after tubal sterilization have been challenged by the US Collaborative Review of sterilization, ${ }^{24}$ we tried to assess the ovarian function by studying menstrual cycle irregularities 1-2 years after sterilization; we also attempted to address quality of life issues as well as rates of regrets.

Our study has strengths and limitations. The limitations are as follows: 1) our study was performed in a single center; therefore, our results may not be reproducible in other populations; 2) although our sample size is comparable with that of previously reported research in the field, we may not be adequately powered to detect differences in our primary outcomes; and 3) although we did not find a statistical association of bilateral salpingectomy with menstrual irregularities or other secondary outcomes, these results should be treated with caution due to our low response rate and high risk for bias.

Our strengths include as follows: 1) our institution is a referral center that serves a high-risk patient population, with high body mass index and multiple comorbidities. Perhaps, this might be the reason for the higher rate of internal organ injury compared with other studies ${ }^{712-14} ; 2$ ) our study adds clinically relevant data in a less-studied but relevant field; and 3) we obtained results in agreement with previously reported studies, utilizing a technique for total salpingectomy that could be reproduced globally without adding extra cost.

We can conclude that bilateral salpingectomy appears to be a feasible option for sterilization during cesarean delivery in our population, when compared with traditional methods, and should be considered for women interested in ovarian cancer risk-reducing interventions; however, 
we acknowledge the need for multi-center randomized trials to corroborate our outcomes and validate the superiority of bilateral salpingectomy over traditional methods for ovarian cancer prevention.

\section{Ethical statement}

Women included in the secondary outcomes analysis verbally consented to participate in our study. The questionnaire and the study were approved by the University of Tennessee Health Science Center.

\section{Disclosure}

The authors report no conflicts of interest in this work.

\section{References}

1. American Cancer Society. Ovarian Cancer. Located at. Available from: http://www.cancer.org/cancer/ovariancancer/detailedguide/ovariancancer-key-statistics. Accessed October 4, 2018.

2. Kurman RJ, Shih I. Molecular pathogenesis and extraovarian origin of epithelial ovarian cancer - shifting the paradigm. Hum Pathol. 2011; 42(7):918-931.

3. Medeiros F, Muto MG, Lee Y, et al. The tubal fimbria is a preferred site for early adenocarcinoma in women with familial ovarian cancer syndrome. Am J Surg Pathol. 2006;30(2):230-236.

4. Chene G, Rahimi K, Mes-Masson AM, Provencher D. Surgical implications of the potential new tubal pathway for ovarian carcinogenesis. J Minim Invasive Gynecol. 2013;20(2):153-159.

5. Walker JL, Powell CB, Chen LM, et al. Society of Gynecologic Oncology recommendations for the prevention of ovarian cancer. Cancer. 2015; 121(13):2108-2120.

6. Committee on Gynecologic Practice. Committee opinion no. 620: Salpingectomy for ovarian cancer prevention. Obstet Gynecol. 2015;125(1): 279-281.

7. Chan LM, Westhoff CL. Tubal sterilization trends in the United States. Fertil Steril. 2010;94(1):1-6.

8. Cibula D, Widschwendter M, Májek O, Dusek L. Tubal ligation and the risk of ovarian cancer: review and meta-analysis. Hum Reprod Update. 2011;17(1):55-67.

9. Ganer Herman H, Gluck O, Keidar R, et al. Ovarian reserve following cesarean section with salpingectomy vs tubal ligation: a randomized trial. Am J Obstet Gynecol. 2017;217(4):472.
10. Morelli M, Venturella R, Mocciaro R, et al. Prophylactic salpingectomy in premenopausal low-risk women for ovarian cancer: primum non nocere. Gynecol Oncol. 2013;129(3):448-451.

11. Duncan JR, Schenone MH, Mari G. Technique for bilateral salpingectomy at the time of Cesarean delivery: a case series. Contraception. 2017;95(5):509-511.

12. Committee on Practice Bulletins-Obstetrics. Practice Bulletin No. 183 : Postpartum hemorrhage. Obstet Gynecol. 2017;130(4):e168-e186.

13. Powell CB, Alabaster A, Simmons S, et al. Salpingectomy for sterilization: change in practice in a large integrated health care system, 2011-2016. Obstet Gynecol. 2017;130(5):961-967.

14. Subramaniam A, Blanchard CT, Erickson BK, et al. Feasibility of complete salpingectomy compared with standard postpartum tubal ligation at cesarean delivery: a randomized controlled trial. Obstet Gynecol. 2018;132(1):20-27.

15. Garcia C, Moskowitz OM, Chisholm CA, et al. Salpingectomy compared with tubal ligation at cesarean delivery: a randomized controlled trial. Obstet Gynecol. 2018;132(1):29-34.

16. Shinar S, Blecher Y, Alpern S, et al. Total bilateral salpingectomy versus partial bilateral salpingectomy for permanent sterilization during cesarean delivery. Arch Gynecol Obstet. 2017;295(5):1185-1189.

17. Mcalpine JN, Hanley GE, Woo MM, et al. Opportunistic salpingectomy: uptake, risks, and complications of a regional initiative for ovarian cancer prevention. Am J Obstet Gynecol. 2014;210(5):471 e1-e11.

18. Danis RB, Della Badia CR, Richard SD. Postpartum permanent sterilization: could bilateral salpingectomy replace bilateral tubal ligation? J Minim Invasive Gynecol. 2016;23(6):928-932.

19. Ye XP, Yang YZ, Sun XX. A retrospective analysis of the effect of salpingectomy on serum antiMüllerian hormone level and ovarian reserve. Am J Obstet Gynecol. 2015;212(1):53 e1-e10.

20. Van Lieshout LAM, Pijlman B, Vos MC, et al. Opportunistic salpingectomy in women undergoing hysterectomy: results from the HYSTUB randomised controlled trial. Maturitas. 2018;107:1-6.

21. Song T, Kim MK, Kim ML, et al. Impact of opportunistic salpingectomy on anti-Müllerian hormone in patients undergoing laparoscopic hysterectomy: a multicentre randomised controlled trial. $B J O G$ 2017;124(2):314-320.

22. Mohamed AA, Yosef AH, James C, et al. Ovarian reserve after salpingectomy: a systematic review and meta-analysis. Acta Obstet Gynecol Scand. 2017;96(7):795-803.

23. Venturella R, Morelli M, Lico D, et al. Wide excision of soft tissues adjacent to the ovary and fallopian tube does not impair the ovarian reserve in women undergoing prophylactic bilateral salpingectomy: results from a randomized, controlled trial. Fertil Steril. 2015;104(5):1332-1339.

24. Peterson HB, Jeng G, Folger SG, et al. The risk of menstrual abnormalities after tubal sterilization. U.S. Collaborative Review of Sterilization Working Group. N Engl J Med. 2000;343(23):1681-1687.
International Journal of Women's Health

\section{Publish your work in this journal}

The International Journal of Women's Health is an international, peerreviewed open-access journal publishing original research, reports, editorials, reviews and commentaries on all aspects of women's healthcare including gynecology, obstetrics, and breast cancer. The manuscript management system is completely online and includes

\section{Dovepress}

a very quick and fair peer-review system, which is all easy to use. Visit http://www.dovepress.com/testimonials.php to read real quotes from published authors. 